(randomly selected youths). Exposures of interest were collected for the months prior to injury events for cases and randomly selected months for controls, based on an injury incidence algorithm. Multivariate logistic regression characterised associations between specific animal-related activities and injury outcomes among youths who reported working with the same animals.

Results A large proportion of cases and controls worked with beef cattle $(47 \%, 28 \%)$, followed by horses $(28 \%, 14 \%)$ and dairy cattle $(22 \%, 12 \%)$. Feeding was the most common activity associated with animals in the study. Among those working with swine, $100 \%$ of cases (reporting swine-related injuries) and $85 \%$ of controls indicated feeding these animals during the month of interest. Activities most reported for dairy cows and beef cattle, respectively, were milking (63\%, 44\%) and herding $(81 \%, 61 \%)$. Elevated risk of dairy cattle-related injury was associated with milking (OR: 2.5; 95\% CI 1.0 to 6.6). Beef cattle-related injuries were associated with calving (OR: 4.2; 95\% CI 2.1 to 8.6) and footwork (OR: 2.2; 95\% CI 1.0 to 4.9 ).

Conclusions Among youths working with a specific type of animal, activities can be identified that are associated with injuries related to that animal. Identifying specific hazardous tasks is necessary for effective prevention measures.

\title{
0576 ANIMAL-RELATED ACTIVITIES AS RISK FACTORS FOR INJURIES AMONG YOUTHS ON AGRICULTURAL OPERATIONS
}

A D Ryan*, S G Gerberich, B H Alexander, C M Renier Correspondence: , Division of Environmental Health Sciences School of Public Health, University of Minnesota, School of Public Health, 1260 Mayo Memorial Building, 420 Delaware Street, SE Minneapolis, MN 55455, USA

\subsection{6/ip.2010.029215.576}

Introduction Working with animals on agricultural operations is hazardous for youths. We evaluated the associations between activities and injuries related to specific animal types.

Methods A case-control study within the Regional Rural Injury Study II included 425 youths (less than 20 years of age) with injuries related to their operation and 1886 controls 